\title{
Dye-Specific Wavelength Offsets to Resolve Spectrally Overlapping and Co-Localized Two-Photon Induced
}

\section{Fluorescence}

C. Kyle Almlie, Austen Hsiao, and Sean M. Burrows*

Oregon State University, Department of Chemistry, Analytical Division, 153 Gilbert Hall, Corvallis, Or 97331.

Email: Sean.Burrows@oregonstate.edu

\begin{abstract}
General methods and procedures to scan through dye-specific offsets are covered. Data processing to obtain excitation spectra and excitation-emission spectral matrices are described in detail. Results from constant-offset, variable-angle, and non-linear variable-angle (NLVA) offset scans are presented and discussed. Tables for cross-talk percentage point changes from increasing and decreasing the $\mathrm{RhB}$ concentration are presented here.
\end{abstract}


Methods:

Two-Photon Dye-Specific Offset (TP-DSO) Scanning Procedure:

A spectrum was acquired by a manual process consisting of the following sequence:

1. The desired excitation wavelength was set and the power adjusted to $100 \mathrm{~mW}$ using a half-wave plate and polarizer.

2. The LVF's were set to their respective positions to form the desired bandpass centered at the emission wavelength specified by the dye-specific offset (DSO).

3. An Arduino-controlled beam block was opened to illuminate the sample. The beam block was positioned after the beam sampler in the beam path to prevent interruption of laser power measurements.

4. A spectrum was collected with the QE65Pro spectrometer.

5. The beam block was closed after a spectrum was collected to prevent photobleaching of the sample between sequences.

6. Steps $1-5$ were repeated until spectra from all dye-specific offsets and bandwidths was collected.

Supplementary Figures, Discussions, and Tables:
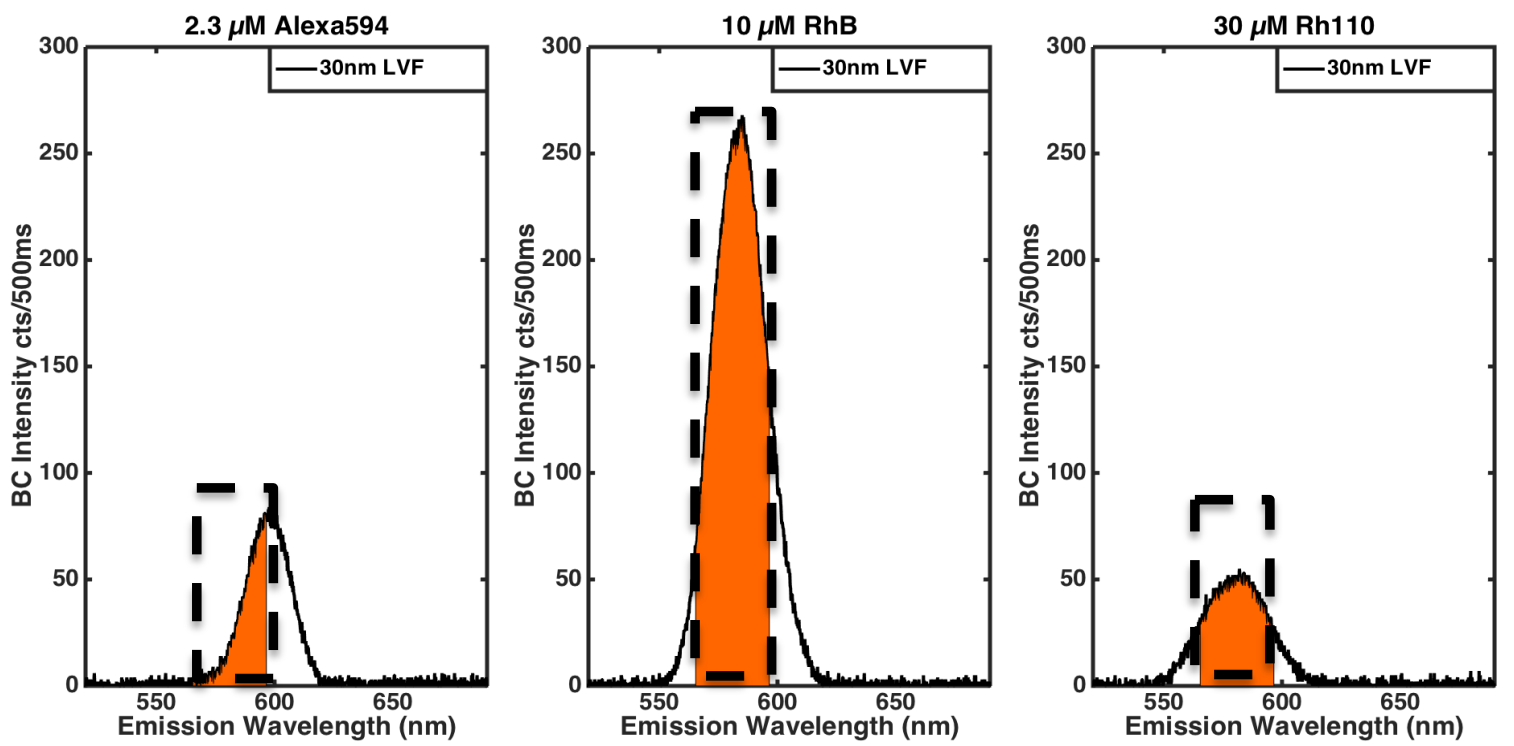
Figure S1 - Shows an example of how intensity and cross-talk contribution was determined. For this example the RhB dye-specific offset (820/580) with a $30 \mathrm{~nm}$ LVF bandwidth was used. The intensity from each dye was summed from 565 to $595 \mathrm{~nm}$ (180 pixels, dashed box). The summed signal intensity is shown as the orange areas under the peak. Note the summed area of the Alexa594 peak for the RhB DSO does not appear to include the full Alexa594 peak. This is because the full Alexa594 emission peak is stronger in the $600 \mathrm{~nm}$ area than RhB. However, the pixels that defined the DSO were still summed as shown by the dashed box.
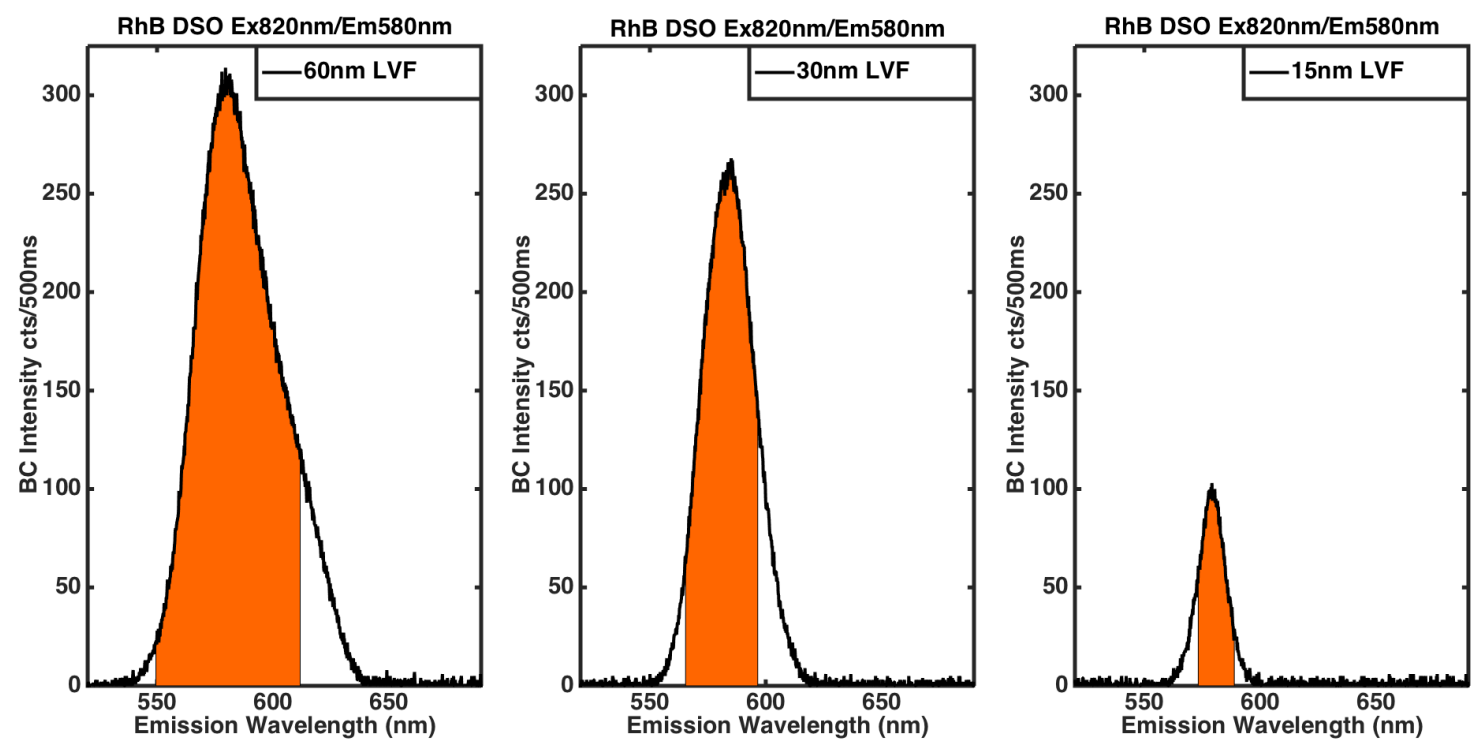

Figure S2 - From left to right $60 \mathrm{~nm}, 30 \mathrm{~nm}$, and $15 \mathrm{~nm}$ emission peaks formed by LVFs centered at 580 $\mathrm{nm}$ for $10 \mu \mathrm{M} \mathrm{RhB}$ in the RhB excited at $820 \mathrm{~nm}$. The orange areas represent the summed signal intensity for the given bandpass. The $60 \mathrm{~nm}$ bandpass is summed over $60 \mathrm{~nm}$ (360 pixels), the $30 \mathrm{~nm}$ bandpass is summed over $30 \mathrm{~nm}$ (180 pixels), and the $15 \mathrm{~nm}$ bandpass is summed over $15 \mathrm{~nm}$ (90 pixels).

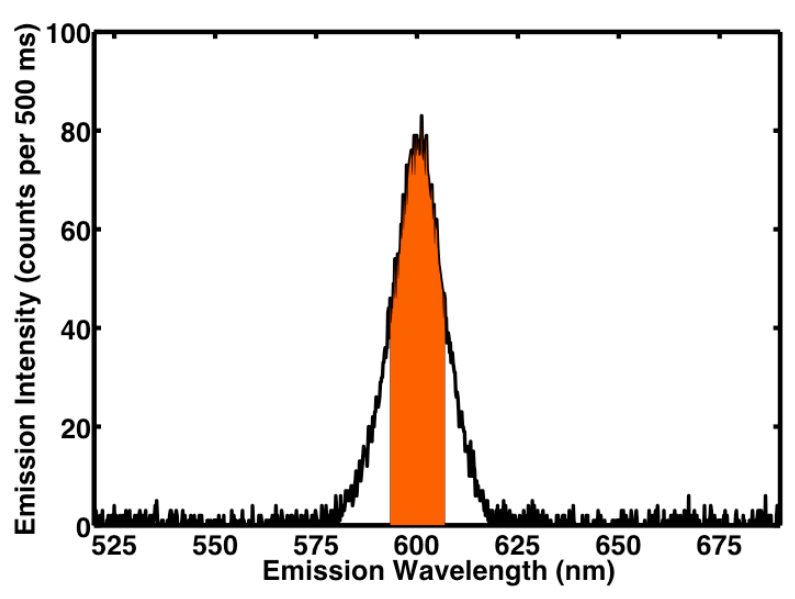


Figure S3. Representative RhB emission spectra (LVF with a $15 \mathrm{~nm}$ bandpass) used to calculate the data points for the constant-offset excitation spectra shown in main text Figures 3 (A - C, colored diamonds) and Figures S5 (black triangles (top) colored markers (bottom)) and S6 (colored markers). Data points for their respective spectrum were the summed intensity of 40 pixels on either side of the DSO emission peak wavelength formed by the LVFs ( $\sim 15 \mathrm{~nm}$ FWHM, orange-shaded area).

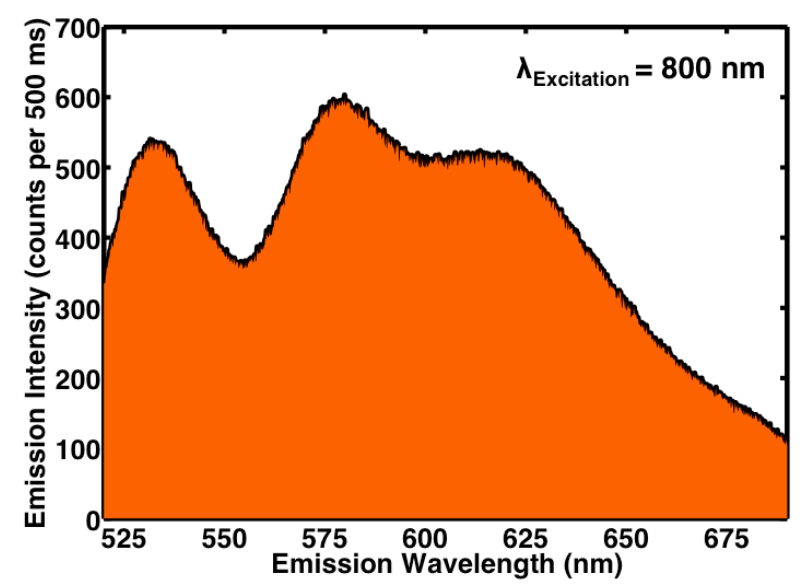

Figure S4. Representative emission spectra (no linear variable filters) of a mixture used to determine the excitation spectra shown in main text Figure 1B (black triangles). Each point in the excitation spectra were obtained by summation of a full emission spectrum (orange-shaded area). Main text Figure 1B (colored markers), Figures 3 (A - C, black triangles), and below in Figure S5 (blue circles (top)) were achieved by the same method using their respective solutions. 

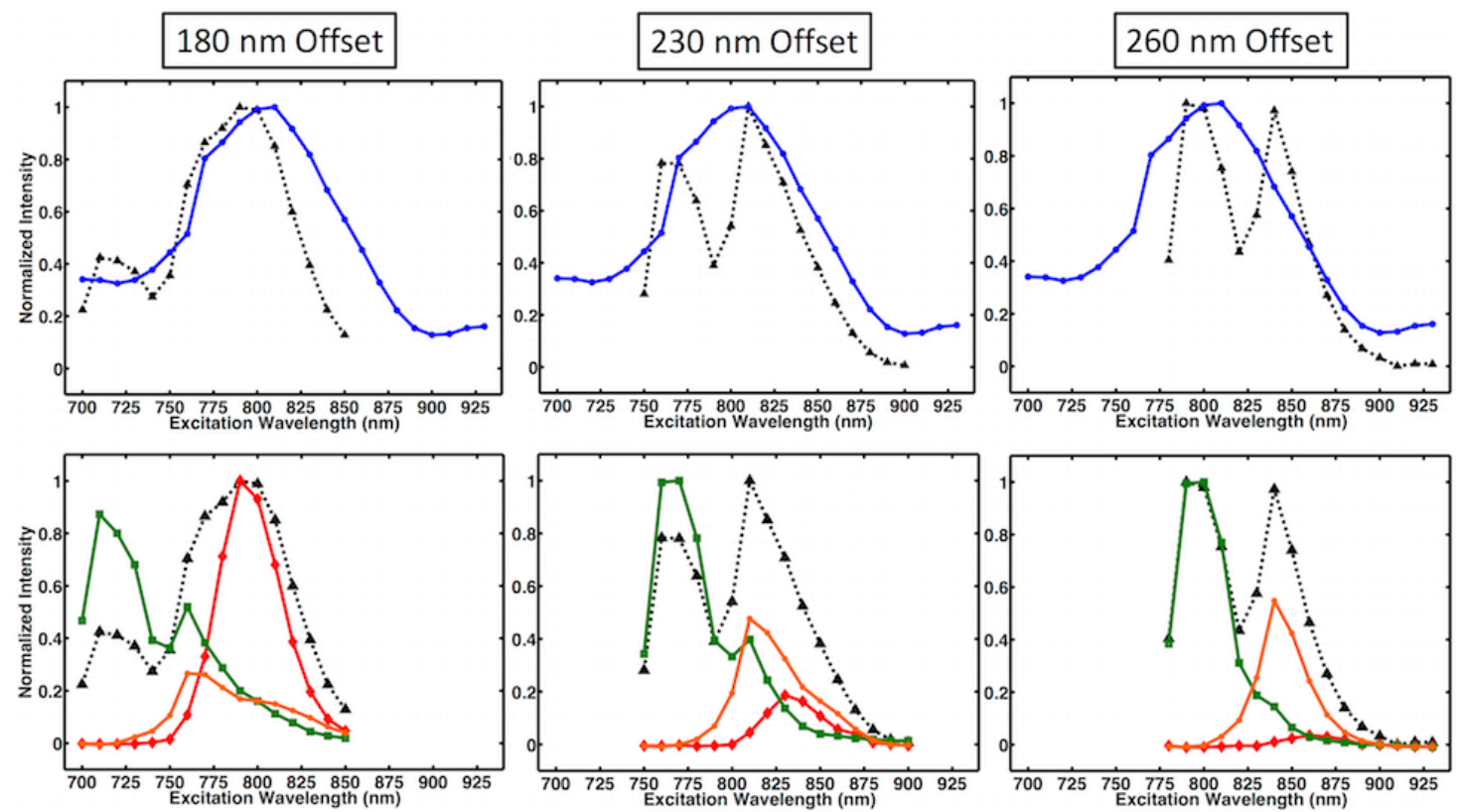

$\because$ A m Mixture
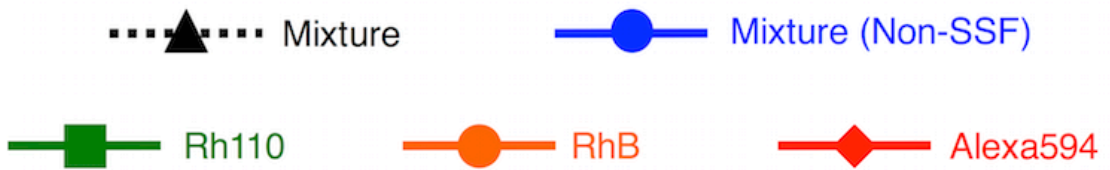

Figure S5. The effects of constant-offset synchronous scanning on a mixture of three dyes. The $180 \mathrm{~nm}$ offset corresponded to the ideal offset for Alexa594, the $260 \mathrm{~nm}$ offset was an average of $250 \mathrm{~nm}$ and $270 \mathrm{~nm}$, the ideal offsets for $\mathrm{RhB}$ and $\mathrm{Rh} 110$, respectively. The $230 \mathrm{~nm}$ offset was the average offset of 180,250 , and $270 \mathrm{~nm}$. The top row shows the two-photon excitation spectrum (no LVF) of the mixture solution (blue circles) and the two-photon synchronous spectrum (TP-SS) of the mixture (black triangles) at each offset indicated. The bottom row has the same TP-SS of the mixture (black triangles) as the top row, but with the TP-SS of the individual dyes as controls at each constant-offset indicated (Rh110 = green squares, $\mathrm{RhB}=$ orange circles, and Alexa594 = red diamonds).

The figure shows that a constant-offset scan near a given dyes offset (that was equal to the difference of excitation and emission peaks) will have selectivity for that dye. Each constant-offset TP-SS only resolved two dyes at a time; however, the cross-talk from both RhB and Alexa594 in the Rh110 emission region was always decreased for each constant-offset scan. For example, in the bottom figure for the 260 $\mathrm{nm}$ offset column, the Alexa594 cross-talk was reduced from 4 to $2 \%$ upon use of TP-SS, but the RhB remained an issue over the 775 to $825 \mathrm{~nm}$ region. Additionally, we observed about $26 \%$ cross-talk of the Rh110 peak at the RhB peak DSO $(840 / 580 \mathrm{~nm})$, which was an increase from $18 \%$ in the no-LVF control. At the $260 \mathrm{~nm}$ offset, the emission from both Rh110 and RhB was very strong and Rh110 bled into the RhB channel, while Alexa594's was not selected because its dye-specific offset $(180 \mathrm{~nm})$ was far away from the offset being scanned $(260 \mathrm{~nm})$. As a result, Alexa594 had little contribution to cross-talk 
for the $260 \mathrm{~nm}$ scan. Similarly, the $230 \mathrm{~nm}$ offset performed poorly because the dyes were approximately equidistant from their dye-specific offset, reducing the effectiveness of these TP-DSOs.

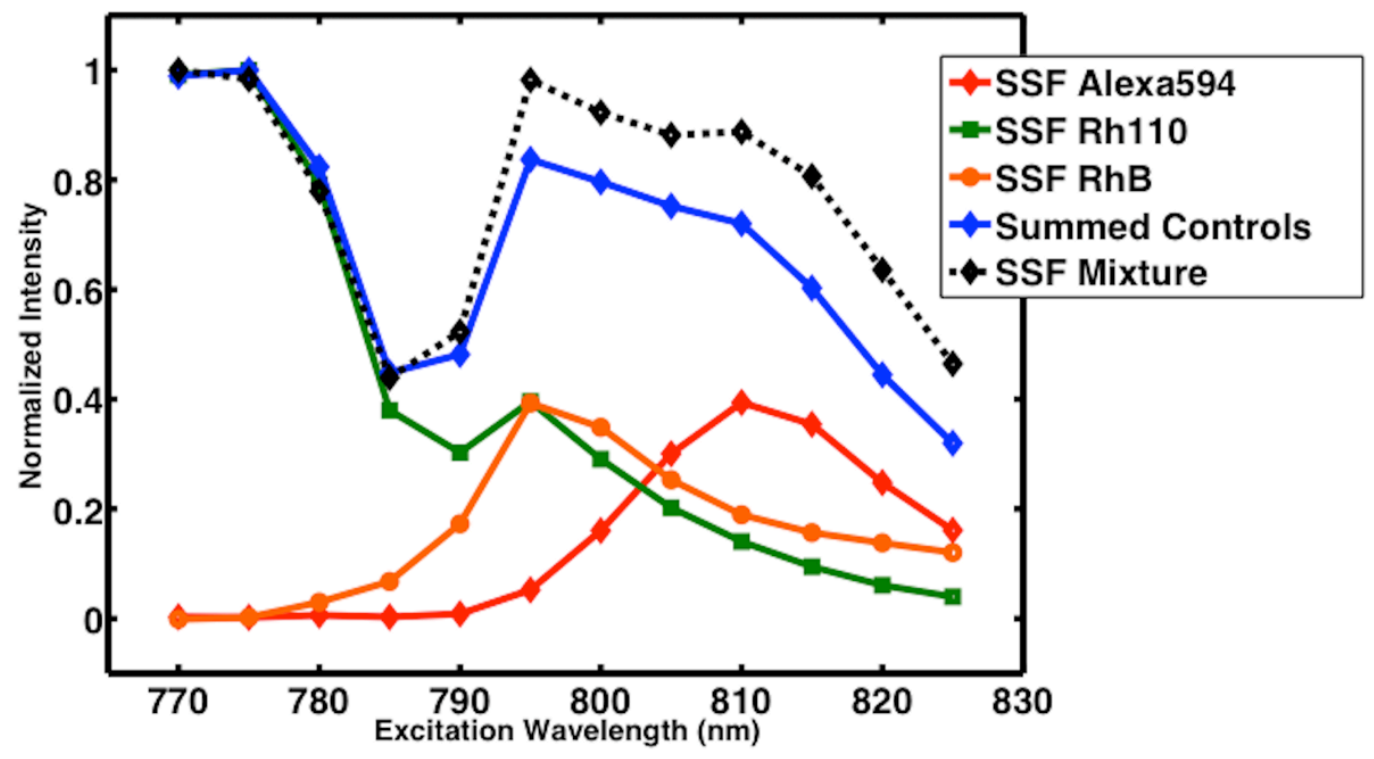

Figure S6. Variable-angle offset TP-synchronous spectrum (TP-SS) with a mixture and individual solutions of three dyes. The offset decreased by $5 \mathrm{~nm}$ for every $10 \mathrm{~nm}$ increase of the emission wavelength. At $775 \mathrm{~nm}$ excitation the offset was $235 \mathrm{~nm}$ (emission $=540 \mathrm{~nm}$ ). At $795 \mathrm{~nm}$ excitation, the offset was $215 \mathrm{~nm}($ emission $=580 \mathrm{~nm})$, and at $810 \mathrm{~nm}$ excitation the offset was $200 \mathrm{~nm}($ emission $=610$ $\mathrm{nm}$ ). While variable-angle offset attempted to achieve the dye-specific offset for each dye during a single scan, it was not able to reduce cross-talk between all three dyes in solution. For example, the $215 \mathrm{~nm}$ offset attempted to select for $\mathrm{RhB}(795 / 580 \mathrm{~nm})$; but variable-angle scanning lead to nearly $100 \%$ overlap with Rh110. In this case, approximating the RhB DSO with variable-angle scanning did not reduce cross-talk since both dyes were excited at $795 \mathrm{~nm}$ and emitted approximately equal intensities at $580 \mathrm{~nm}$ (using LVFs). Therefore, beyond $780 \mathrm{~nm}$ excitation, the resulting TP-SS was an overlap of emission from all three dyes. 

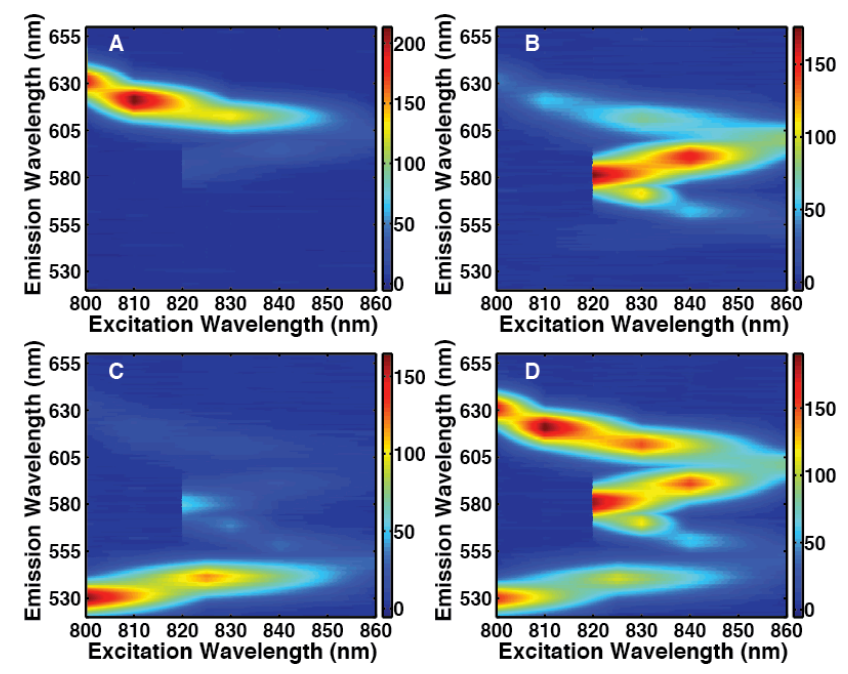

Figure S7. Non-Linear Variable-Angle TP-synchronous scans (NLVA TP-SS) of (A) Alexa594, (B) RhB, (C) Rh110. and (D) a mixture of all three dyes. Intensity bar in units of counts per $500 \mathrm{~ms}$. This figure gives visual spectral resolution along the excitation and emission axis. Dye-specific offsets were developed from this figure and Figure S6, where the peaks of maximum signal (deep red) from each dye (A-C) were selected for the dye-specific offsets.

Table S1. Reduction (percentage point) of cross-talk with LVF and dye-specific offsets with $5 \mu \mathrm{M} \mathrm{RhB}$, $2.3 \mu \mathrm{M}$ Alexa594, and $30 \mu \mathrm{M}$ Rh110.

\begin{tabular}{|c|c|c|c|c|c|c|}
\hline \multirow{2}{*}{$\begin{array}{l}\text { Dye-Specific Offset } \\
\text { (Ex/Em, nm) }\end{array}$} & \multicolumn{3}{|c|}{$\begin{array}{l}\text { Dyes with } 15 \mathrm{~nm} \text { LVF bandpass } \\
(\mathrm{n}=90 \text { pixels summed })\end{array}$} & \multicolumn{3}{|c|}{$\begin{array}{l}\text { Dyes with } 30 \mathrm{~nm} \text { LVF bandpass } \\
(\mathrm{n}=180 \text { pixels summed })\end{array}$} \\
\hline & \begin{tabular}{|l} 
(Alexa594) \\
\end{tabular} & (RhB) & (Alexa594) & (RhB) & (Alexa594) & (RhB) \\
\hline Alexa594: $(810 / 620)$ & - & 4.27 & 3.40 & - & 4.35 & 3.17 \\
\hline Alexa594: $(820 / 630)$ & - & -1.17 & $0.63^{*}$ & 0 & $-0.04 *$ & 0.87 \\
\hline RhB: $(820 / 580)$ & 62.57 & - & 49 & 42.66 & - & 27.52 \\
\hline $\mathrm{RhB}$ & 51.26 & - & 66.47 & 37 & - & 24.62 \\
\hline Rh110: $(800 / 530)$ & $-0.69 *$ & 4.71 & - & $0.16^{*}$ & 5.07 & - \\
\hline \multicolumn{7}{|c|}{$\begin{array}{l}(\alpha=0.01, \mathrm{n}=360 \text { and } 180 \text { or } 90),{ }^{*} \text { no statistical difference. } \\
\text { Positive values indicate the percent points cross-talk was reduced compared to the } 60 \mathrm{~nm} \text { bandpass usec } \\
\text { for the conventional filter model. A dash indicates that the cross-talk was not applicable because the } \\
\text { emission was normalized to that DSO. }\end{array}$} \\
\hline
\end{tabular}

Table S2. Reduction (percentage point) of cross-talk with LVF and dye-specific offsets with $20 \mu \mathrm{M} \mathrm{RhB}$, $2.3 \mu \mathrm{M}$ Alexa594, and $30 \mu \mathrm{M}$ Rh110.

\begin{tabular}{|c|c|c|} 
Dye-Specific Offset & $\begin{array}{c}\text { Dyes with } 15 \mathrm{~nm} \text { LVF bandpass } \\
(\mathrm{n}=45 \text { pixels summed })\end{array}$ & $\begin{array}{c}\text { Dyes with } 30 \mathrm{~nm} \text { LVF bandpass } \\
(\mathrm{n}=90 \text { pixels summed })\end{array}$ \\
\hline
\end{tabular}




\begin{tabular}{|c|c|c|c|c|c|c|}
\hline$(\mathrm{Ex} / \mathrm{Em}, \mathrm{nm})$ & (Alexa594) & $(\mathrm{RhB})$ & (Alexa594) & $(\mathrm{RhB})$ & (Alexa594) & $(\mathrm{RhB})$ \\
\hline Alexa594: $(810 / 620)$ & - & 25.15 & 3.40 & - & 24.16 & 3.17 \\
\hline Alexa594: (820/630) & - & $5.08 * *$ & $0.63 *$ & - & $5.31 * *$ & 0.87 \\
\hline RhB: $(820 / 580)$ & 14.69 & - & 11.70 & 10.28 & - & 6.90 \\
\hline RhB: $(710 / 580)$ & 10.74 & - & 13.97 & 7.76 & - & 0.87 \\
\hline Rh110: $(800 / 530)$ & $-0.69 *$ & 18.21 & - & $0.16^{*}$ & 17.69 & - \\
\hline \multicolumn{7}{|c|}{$\begin{array}{l}(\alpha=0.01, \mathrm{n}=360 \text { and } 180 \text { or } 90), * \text { no statistical difference, } * * \text { cross talk was statistically reduced, but } \\
\text { remained }>100 \% \text {. } \\
\text { Positive values indicate the percent points cross-talk was reduced compared to the } 60 \mathrm{~nm} \text { bandpass used } \\
\text { for the conventional filter model. A dash indicates that the cross-talk was not applicable because the } \\
\text { emission was normalized to that DSO. }\end{array}$} \\
\hline
\end{tabular}

